\title{
The Impact of Resolution on a Numerical Simulation of Barotropic Instability*
}

\author{
AMY SOLOMON \\ International Pacific Research Center, School of Ocean and Earth Science and Technology, \\ University of Hawaii at Manoa, Honolulu, Hawaii \\ RICHARD S. LINDZEN \\ Department of Earth, Atmospheric and Planetary Sciences, Massachusetts Institute of Technology, \\ Cambridge, Massachusetts
}

(Manuscript received 15 March 1999, in final form 27 January 2000)

\section{ABSTRACT}

\begin{abstract}
The barotropic point jet problem is used to study the impact of resolution on a numerical simulation of barotropic instability. This particular problem is studied because of the close relation of the linearized version of the problem to baroclinic instability.

This study finds that the channel-averaged wave enstrophy and the fluxes at the jet in the wave-mean flow equilibrated state are underestimated when the resolution of the model is inadequate to resolve the analytic linear growth rates. Moreover, the resolution of the model is found to impact the wave-mean flow equilibrated state even when the analytic linear growth rates are resolved. This is due to potential vorticity gradients and fluxes in the equilibrated state being largely independent of resolution, as long as the linear growth rates are adequately resolved. In a coarse-resolution model this results in momentum fluxes and shear at the vertex of the jet that are dependent upon the resolution of the model.

The results of this study suggest that resolution will also impact the numerical simulation of baroclinic instability.
\end{abstract}

\section{Introduction}

Two-level models of the atmosphere are commonly used to study the large-scale processes of the midlatitude troposphere. The eddies in the two-level model studies act to bring the model's mean state to an equilibrium that has meridional temperature gradients that are smaller than the temperature gradients in radiative-convective equilibrium. These results have been used to support the hypothesis that dynamical heat transports due to baroclinic eddies play a dominant role in determining the temperature structure of the midlatitude troposphere. Stone (1978) proposed that baroclinic eddies that have been destabilized by a surface temperature gradient will act to reduce the temperature gradient in order to stabilize the eddies. By the thermal wind relation, a me-

* School of Ocean and Earth Science and Technology Contribution Number 5003 and International Pacific Research Center Contribution Number 27.

Corresponding author address: Dr. Amy Solomon, International Pacific Research Center, SOEST, University of Hawaii, 1000 Pope Road, Honolulu, HI 96822.

E-mail: amy@iprc.soest.hawaii.edu ridional temperature gradient is proportional to the zonal wind shear. Therefore, the critical temperature gradient needed to stabilize the flow can be expressed as a critical zonal wind shear. Stone (1978) proposed that this critical shear was equivalent to the critical wind shear for instability in the two-level quasigeostrophic model, derived by Phillips (1954). Stone used the term "baroclinic adjustment" for the process of stabilizing the flow by dynamical heat transports. He found a strong coincidence between Phillips's critical shear and the observed shear in the midtroposphere. This coincidence was found for all seasons, regardless of the large seasonal variations in the diabatic forcing. This was earlier noted by Pocinki and reported in Thompson (1961, p. 113).

Stone and Branscome (1992) studied large-scale eddies in a two-level quasigeostrophic midlatitude $\beta$-plane model. In this model study the static stability was set equal to a constant, so the change in the temperature structure due to baroclinic adjustment was characterized by the steady-state meridional temperature gradient. The results of the study showed that the shear of the steady state was proportional, and supercritical, to Phillips's two-level critical shear. The results of the Stone and Branscome study imply that a weaker form of the baroclinic adjustment hypothesis has a strong influence in determining the equilibrium temperature structure. A 
further result of this study was that the meridional temperature gradient was independent of the magnitude of the diabatic forcing and linearly proportional to the static stability. These results are consistent with the baroclinic adjustment hypothesis and with the neutral state predicted by the Charney-Stern criterion for stability. The main difference between the results of this study and the predicted neutral state is that the equilibrium shear is still unstable by the Charney-Stern theorem, even though the growth of the baroclinic eddies has been suppressed in the model.

The Charney-Stern criterion for stability requires that, in the absence of surface temperature gradients, the meridional potential vorticity gradient must not change sign. In the two-level model with fixed static stability, this criterion is equivalent to Phillips's critical shear (viz. Lindzen 1993). In the two-level model with variable static stability, both the static stability and the zonal wind shear are modified by the baroclinic adjustment (Held and Suarez 1978; Held 1978b; Zhou and Stone 1993). In a continuous model, the vertical profile and the magnitude of the zonal wind and/or the static stability are modified by the baroclinic adjustment (Lindzen and Farrell 1980b; Gutowski 1985; Gutowski et al. 1989). The extreme vertical truncation of the twolevel model makes it unclear how baroclinic adjustment will be realized in a model with more detailed vertical structure.

The present paper considers the role of resolution on a simpler but related problem: namely, the nonlinear evolution of a barotropic point jet whose linear stability properties are identical to those of the Boussinesq Charney problem (Lindzen et al. 1983). The cross-stream direction in the barotropic problem corresponds to the vertical direction in the baroclinic problem. This model study focuses on the modification of eddy-mean flow interactions due to variations in the "vertical" resolution. While the barotropic point jet cannot be used to investigate the impact of resolution on the full threedimensional baroclinic problem (e.g., the barotropic point jet cannot be used to determine the importance of feedbacks associated with a variable static stability or feedbacks associated with barotropic effects on baroclinic instability), these results may suggest to what extent the results of two-level model studies, such as Stone and Branscome (1992), Zhou and Stone (1993), and Held (1978b), can be extended to a multilevel model and to what extent the results are determined by the extreme truncation of a two-level model.

Section 2 describes the model used in this study and discusses previous studies of the barotropic point jet. Section 3 discusses the results of the linearized version of the model. Section 4 discusses the modification to the results of section 3 when wave-mean flow interactions are allowed. Section 5 explores the extension of the results of section 4 to the baroclinic problem. Section 6 presents a brief summary of the results of this study of the barotropic point jet.

\section{The barotropic point jet}

The barotropic point jet problem was developed for a number of reasons including the possibility of providing a simplified context for examining the nonlinear equilibration of an instability analogous to baroclinic instability. Lindzen et al. (1983) showed that the barotropic easterly jet stability problem is mathematically equivalent to the linear Boussinesq Charney problem. The linearized equation for the time rate of change of the perturbation potential vorticity, $q^{\prime}$, on a basic zonal flow, is

$$
\frac{\partial q^{\prime}}{\partial t}+\bar{u} \frac{\partial q^{\prime}}{\partial x}+v^{\prime} \frac{\partial \bar{q}}{\partial y}=-D q^{\prime}
$$

where the meridional potential vorticity gradient is

$$
\frac{\partial \bar{q}}{\partial y}=\beta-\bar{u}_{y y}
$$

and $D^{-1}$ is the dissipation time constant and $u$ and $v$ are components in $x$ and $y$ directions. In the wave-mean flow integrations, to be discussed in section 4 , this term acts to both dissipate the waves and maintain the mean flow. In the barotropic problem, there is no distinction between perturbation potential vorticity and perturbation vorticity,

$$
q^{\prime}=\frac{\partial^{2} \psi}{\partial y^{2}}+\frac{\partial^{2} \psi}{\partial x^{2}},
$$

where $\psi$ is the perturbation streamfunction. Assuming normal-mode solutions, the equation governing the linearized perturbations reduces to

$$
\psi_{y y}+\left[\frac{\beta-\bar{u}_{y y}}{\bar{u}-c}-k^{2}\right] \psi=i D \frac{\left(-k^{2} \psi+\psi_{y y}\right)}{k(\bar{u}-c)},
$$

where solutions are assumed to be of the form $\Psi=$ $\psi(y) e^{i k(x-c t)}$.

Figure 1 shows the initial jet structure and the potential vorticity gradient of the basic state for the barotropic half jet and for the Charney problem. In a numerical simulation of this problem, Schoeberl and Lindzen (1984) demonstrated that in the absence of forcing, an unstable jet evolved to a steady state that was neutral to the eddies. This state was the neutral state as defined by the Charney-Stern criterion for stability. The shear of the flow was modified by the waves as predicted by the linear adjustment theory. The jet evolved from a point to a parabola such that

$$
\bar{q}_{y}=\beta-\bar{u}_{y y}=0 \text {. }
$$

When dissipation was added to the system, the neutral state was found to be supercritical by the Charney-Stern theorem, even though the growth of the waves had been suppressed. The steady state in the presence of dissipation was found to be independent of the magnitude of the dissipation. The supercritical neutral state was found to have a balance between the generation of vor- 


\section{Multi-level Model Profiles}

\section{Barotropic \\ Zonal Mean Wind \\ PV Gradients}

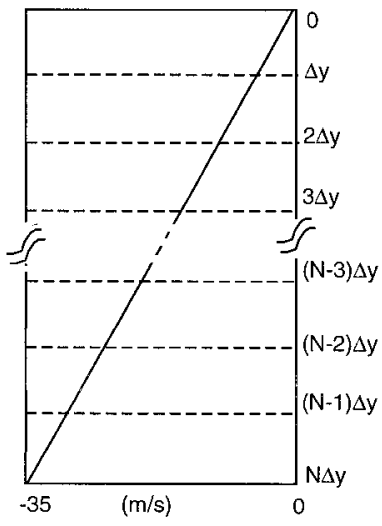

Baroclinic

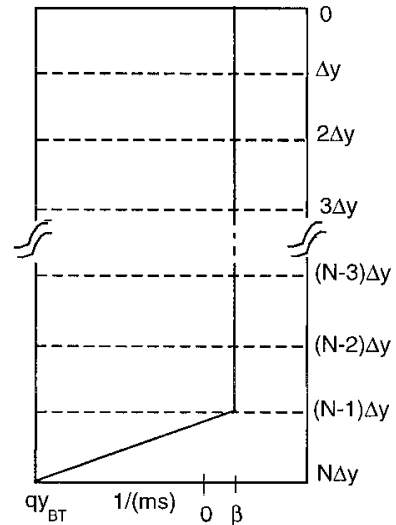

PV Gradients
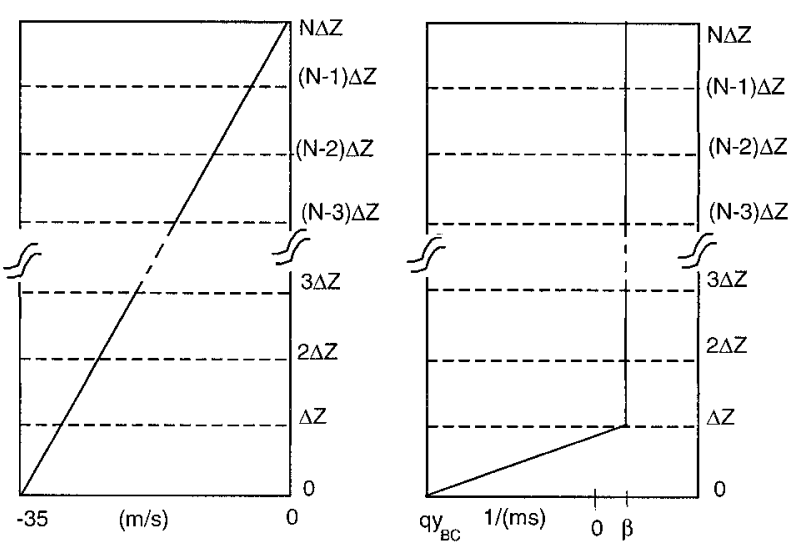

FIG. 1. The initial jet structure and the potential vorticity gradien of the basic state for the barotropic jet and for the Charney problem. The $q y_{B T}$ and $q y_{B C}$ signify the meridional potential vorticity gradients at the boundary in the barotropic jet and the baroclinic Charney problem, respectively.

ticity by the mean flow and the dissipation of vorticity due to the eddies. Schoeberl and Lindzen (1984) note that the neutral state would not be independent of the magnitude of the dissipation if the same linear damping had not been used for both perturbations and the maintenance of the mean flow.

The present problem allows us to investigate the primary aspects of the impact of resolution on the linearized Charney problem in a two-dimensional model. The dispersion relation, relating growth rate, $\beta$, zonal wind, and resolution, is easily obtained.

Since the barotropic jet is two-dimensional it cannot be used to investigate the full three-dimensional nonlinear baroclinic problem. The analogy between the linearized barotropic jet and the linearized Boussinesq Charney problem assumes that the meridional wavenumber is equal to zero. Even though this is the most unstable wavenumber for the Charney problem, it is most likely not the dominant meridional wavenumber that would be found in the presence of a jet. Lindzen (1993) notes that a meridional jet of width $L$ would impose a meridional wavenumber of $l=1 / L$ on the baroclinic instabilities. For a characteristic winter subtropical jet with a width of $0.15 a$, where $a$ is the radius of the earth, this meridional wavenumber is found to be close to global wavenumber 6 . Observations of the midlatitude winter indicate that this is the wavenumber associated with the maximum normal-mode growth. Solving the linearized Boussinesq Charney problem in a channel will also introduce a meridional scale.

\section{Linear results}

In order to derive boundary conditions for the barotropic jet that are analogous to the Charney problem, symmetry of the perturbation streamfunction was required at the vertex of the jet. Schoeberl and Lindzen (1984), Schoeberl and Nielsen (1986), and Nielsen and Schoeberl (1984) studied the full 1-point jet and imposed the boundary condition that there be no mass flux into the jet at the walls sufficiently far from the jet maximum. This formulation of the problem does not exclude the asymmetric modes from the solution and therefore does not strictly satisfy the requirement that the solution be symmetric about the jet. This was found to be an adequate formulation to the problem that was investigated by these previous studies because, for the values of the zonal mean wind and $\beta$ that were used, the asymmetric modes were found to be small relative to the symmetric modes. This may not be true generally. Therefore, this study will only look at the half jet so that the symmetric boundary condition is strictly satisfied.

The linearized equation for the perturbation streamfunction, Eq. (4), has been solved numerically as an eigenvalue problem for the phase speed, $c$. In the studies of Schoeberl and Nielsen (1986) and Nielsen and Schoeberl (1984) the barotropic problem is solved numerically with the perturbation and mean streamfunction at the same meridional levels. The mean zonal wind is calculated using a centered finite-difference scheme so that the perturbation streamfunction and the mean zonal wind are placed at the same meridional level. When this numerical scheme is used to set up the two-level formulation of the barotropic problem, the use of the centered finite-difference scheme introduces a computational mode into the equilibrium solution. Therefore, a staggered scheme is used, which places the mean streamfunction at the midlevel so that the mean zonal wind is calculated at that level. The use of this scheme in the numerical integration of the barotropic problem removes the computational mode. The boundary conditions on the perturbation streamfunction are a rigid boundary at $y=L$ and a radiation condition at $y=0$. The radiation condition at $y=0$ is 
TABLE 1. Values of the critical zonal wind for resolutions [points $(\mathrm{pt})]$ used in this study of the barotropic point jet.

\begin{tabular}{ccc}
\hline \hline $\begin{array}{c}n \\
(\mathrm{pt})\end{array}$ & $\begin{array}{c}u_{c} \\
\left(\mathrm{~m} \mathrm{~s}^{-1}\right)\end{array}$ & $\begin{array}{c}\Gamma_{c} \\
\left(10^{-5} \mathrm{~s}^{-1}\right)\end{array}$ \\
\hline 3 & -30.00 & 1.20 \\
5 & -15.00 & 0.60 \\
9 & -7.50 & 0.30 \\
17 & -3.75 & 0.15 \\
\hline
\end{tabular}

$$
\frac{\partial \psi^{\prime}}{\partial t}=\frac{\partial \psi^{\prime}}{\partial y} C
$$

Here $C$ is adjusted so that the initial linear growth rates in the wave-mean flow problem are equal to the linear growth rates from the eigensystem for the phase speed. Periodic boundary conditions are applied in the zonal direction. The rigid boundary condition at $y=L$, the point of the half jet, is equivalent to the requirement that the perturbation streamfunction be symmetric about the vertex of the jet. This is the boundary condition that must be imposed in order to make this barotropic problem analogous to the linearized Charney problem. The barotropic problem which is equivalent to the two-level baroclinic problem has three levels where the perturbation streamfunction is defined. If the numerical problem is solved using adequately high resolution, then the results are independent of the scheme used. In all of the calculations, there are 129 points in the zonal direction and the length of the channel is kept fixed at 40000 $\mathrm{km}$. The width of the half jet is held fixed at $2500 \mathrm{~km}$. In all of the integrations $\beta=1.9 \times 10^{-11} \mathrm{~m}^{-1} \mathrm{~s}^{-1}$.

\section{a. Critical zonal mean wind}

In an undissipated system, there is no normal-mode growth when the Charney-Stern criterion for stability is satisfied. This criterion is met when the meridional potential vorticity gradient at the point jet does not change sign and can be written

$$
\beta-\frac{2 \Gamma}{\Delta y} \geq 0
$$

where $\Gamma$ is the constant zonal mean shear of the basic state and is equal to $\Gamma=-u_{0} L^{-1}$. In this problem $L$ is equal to the width of the half jet and $u_{0}$ is the zonal wind at the vertex of the jet. Equation (7) can be solved for the critical zonal mean wind for instability. Table 1 lists the values of the critical zonal wind for resolutions used in this study.

In Table $1, \Gamma_{c}$ is the critical shear and $n$ is the number of meridional gridpoints that define the half jet. For the same value of the zonal mean wind, an increase in resolution will make the problem more unstable. This is because the delta function in the meridional gradient of the potential vorticity becomes better defined when the resolution is increased. This decreases the value of the potential vorticity gradient at the vertex of the jet. A decrease in resolution can increase the value of the potential vorticity gradient to the point where the waves have been completely stabilized. When $\Delta y \rightarrow 0$, the system is always unstable and $\Gamma_{c} \rightarrow 0$. With the given parameters, the 3-point system, which is equivalent to the two-level model, is stable for all zonal mean winds less than $-30 \mathrm{~m} \mathrm{~s}^{-1}$ in magnitude.

\section{b. Linear parameter study}

The linear stability of the Charney problem was studied by Held (1978a). This study derived the vertical scale height of linear quasigeostrophic waves that were destabilized by surface temperature gradients. This vertical scale height, $h$, is only relevant when $h \ll H$, where $H$ is equal to the geometric height of the model and

$$
h=\frac{f_{0}^{2} \partial \bar{u} / \partial z}{N^{2} \partial \bar{q} / \partial y},
$$

where $N^{2}$ is the Brunt-Väisälä frequency and the potential vorticity gradient is evaluated at the jet. The shear and static stability are constant. The horizontal wavelength of the most unstable wave was derived to be proportional to

$$
\lambda_{h}=N h / f_{0} .
$$

Held (1978a) assumed that the horizontal phase speed of the waves was scaled by $h \partial \bar{u} / \partial z$. When $h$ is the relevant scale height,

$$
\frac{\partial \bar{u}}{\partial z} h=\frac{u_{0}}{H} h \ll u_{0} .
$$

The horizontal phase speed, $c$, is $O\left(u_{0}\right)$ in the barotropic point jet problem (Schoeberl and Lindzen 1984; Nielsen and Schoeberl 1984). Therefore, $c$ will be scaled by $u_{0}$ instead of the scaling used by Held (1978a). Using this scaling, the growth rate of the waves would be $O(k c)$ $=O\left(u_{0} f_{0} / N h\right)$. This scaling analysis can be applied to the barotropic point jet by replacing $-\left(f_{0} / N\right)(\partial / \partial z)$ with $\partial / \partial y$. The meridional scale height of the waves, for the barotropic point jet, is calculated to be

$$
\begin{aligned}
l & =\frac{\partial \bar{u}}{\partial y} / \frac{\partial \bar{q}}{\partial y} \\
& =-\Gamma /\left(\beta-\frac{2 \Gamma}{\Delta y}\right) \\
& =(2(n-1) / L-\beta / \Gamma)^{-1} .
\end{aligned}
$$

Since $2 \Gamma / \Delta y>\beta$ for instability, a decrease in the resolution will increase the scale height of the waves. When $l \gg L$, the scale height of the waves is replaced by $L$. The zonal wavelength of the most unstable wave is proportional to $l$. This means that the most unstable wavelength will increase when the resolution is decreased. The growth rate of the most unstable wave is $O(k c)$ and is proportional to $-u_{0} / l=-L \partial \bar{q} / \partial y$. De- 


\section{nondimensional growth rates}

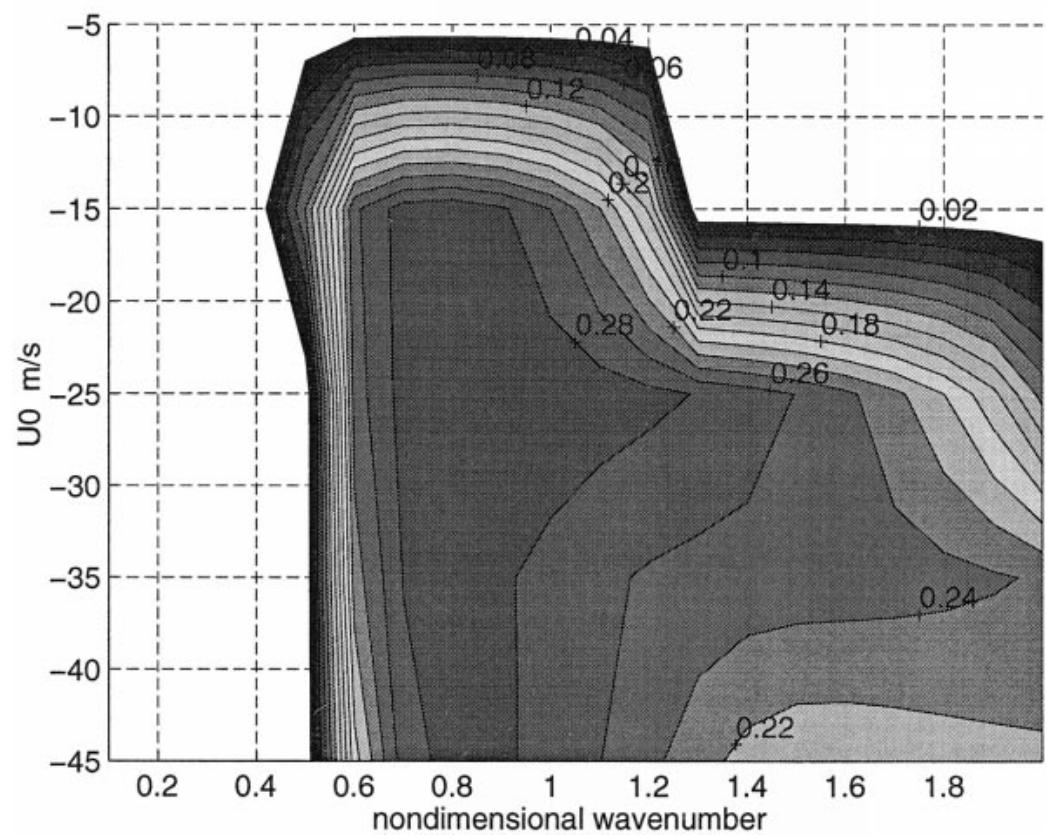

FIG. 2. Nondimensional growth rates when the zonal mean wind is increased from -45 to $-5 \mathrm{~m} \mathrm{~s}^{-1}$, from the model run using the staggered scheme for the 9-point half jet with $1 / D=0$ days and $\beta=1.9 e^{-11} \mathrm{~m}^{-1} \mathrm{~s}^{-1}$.

creasing the resolution decreases the jump in the potential vorticity gradient at the jet and decreases the growth rate of the most unstable wave.

Lindzen and Farrell (1980a) demonstrated that the linearized Boussinesq Charney problem on a semi-infinite plane has a maximum nondimensional growth rate that is independent of $\beta, \Gamma$, and $L$, assuming that the relevant length scale used to nondimensionalize the equations is equal to $\Gamma / \beta$. This maximum nondimensional growth rate is approximately equal to 0.29 and occurs at nondimensional wavenumber 0.9 . The maximum dimensional growth rate is approximately equal to $0.29 \Gamma$ and occurs at wavenumber $0.9 \beta / \Gamma$. Therefore, the maximum dimensional growth rate increases linearly with shear and is independent of $\beta$. This maximum growth rate occurs at longer wavelengths as $\Gamma / \beta$ is increased. Figure 2 shows the nondimensional growth rates when the jet maximum is increased from $-45 \mathrm{~m}$ $\mathrm{s}^{-1}$ to $-5 \mathrm{~m} \mathrm{~s}^{-1}$ in a 9-point model without dissipation, following the scaling of Lindzen and Farrell (1980a). This figure shows that the analytic result, that the maximum nondimensional growth rate is independent of $\Gamma$, breaks down when the zonal mean wind at the jet is less than $-15 \mathrm{~m} \mathrm{~s}^{-1}$ in magnitude. The nondimensional growth rates decrease rapidly as the jet maximum becomes larger than $-15 \mathrm{~m} \mathrm{~s}^{-1}$. All waves become stable for jet maxima less than $-7.5 \mathrm{~m} \mathrm{~s}^{-1}$ in magnitude as predicted by Table 1. Figure 3 shows the dimensional growth rates for the same values of $\beta$ and $\Gamma$ as Fig. 2. This figure shows that the most unstable wave for the 9-point model with a jet maximum of $-35 \mathrm{~m} \mathrm{~s}^{-1}$ without dissipation is a wave 7 with a growth rate of 0.35 day $^{-1}$. Figure 4 shows the nondimensional growth rates for the same parameters as Fig. 2 with a dissipation timescale of $D=0.05$ day $^{-1}$. Comparing Fig. 4 with Fig. 2, it is seen that introducing dissipation causes the nondimensional growth rates to uniformly decrease by approximately 0.04 and stabilizes all waves for a zonal mean wind at the jet less than $-12.5 \mathrm{~m} \mathrm{~s}^{-1}$ in magnitude. The analytical result of a constant nondimensional growth rate breaks down for jet maxima less than $-30 \mathrm{~m} \mathrm{~s}^{-1}$ in magnitude. Figure 5 shows the nondimensional growth rates when $\beta$ is increased from $1.0 \times 10^{-11} \mathrm{~m}^{-1}$ $\mathrm{s}^{-1}$ to $2.6 \times 10^{-11} \mathrm{~m}^{-1} \mathrm{~s}^{-1}$ with a dissipation timescale of 0.05 day $^{-1}$. The maximum nondimensional growth rates begin to decrease for $\beta$ less than $1.0 e^{-11} \mathrm{~m}^{-1} \mathrm{~s}^{-1}$. This is most likely due to poor approximation of a semiinfinite plane when the length scale, $\Gamma / \beta$, becomes very large.

The parameter studies for 5-point resolution are shown in Figs. 6 and 7. Figure 6 shows the nondimensional growth rates when the zonal mean wind is increased from -45 to $-5 \mathrm{~m} \mathrm{~s}^{-1}$. For a zonal mean wind of $-35 \mathrm{~m} \mathrm{~s}^{-1}$, wave 7 is the most unstable wavenumber for the 5-point as well as the 9-point resolution study. For these values of the zonal wind, the reduction in resolution stabilizes both the long waves and the short waves. The nondimensional growth rates decrease as the zonal wind at the jet is increased, becoming neutral for a zonal wind of $-20 \mathrm{~m} \mathrm{~s}^{-1}$. Figure 7 is the 5-point 

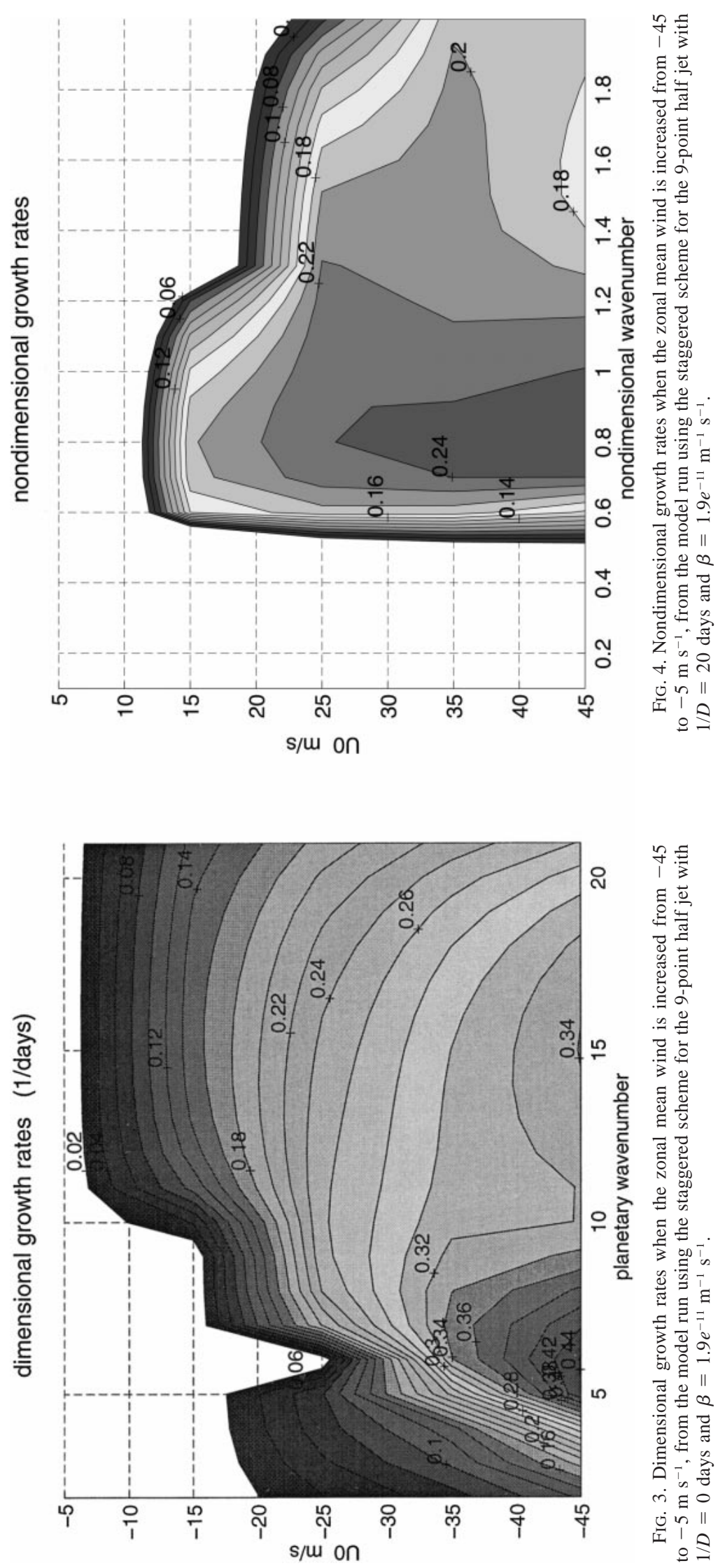


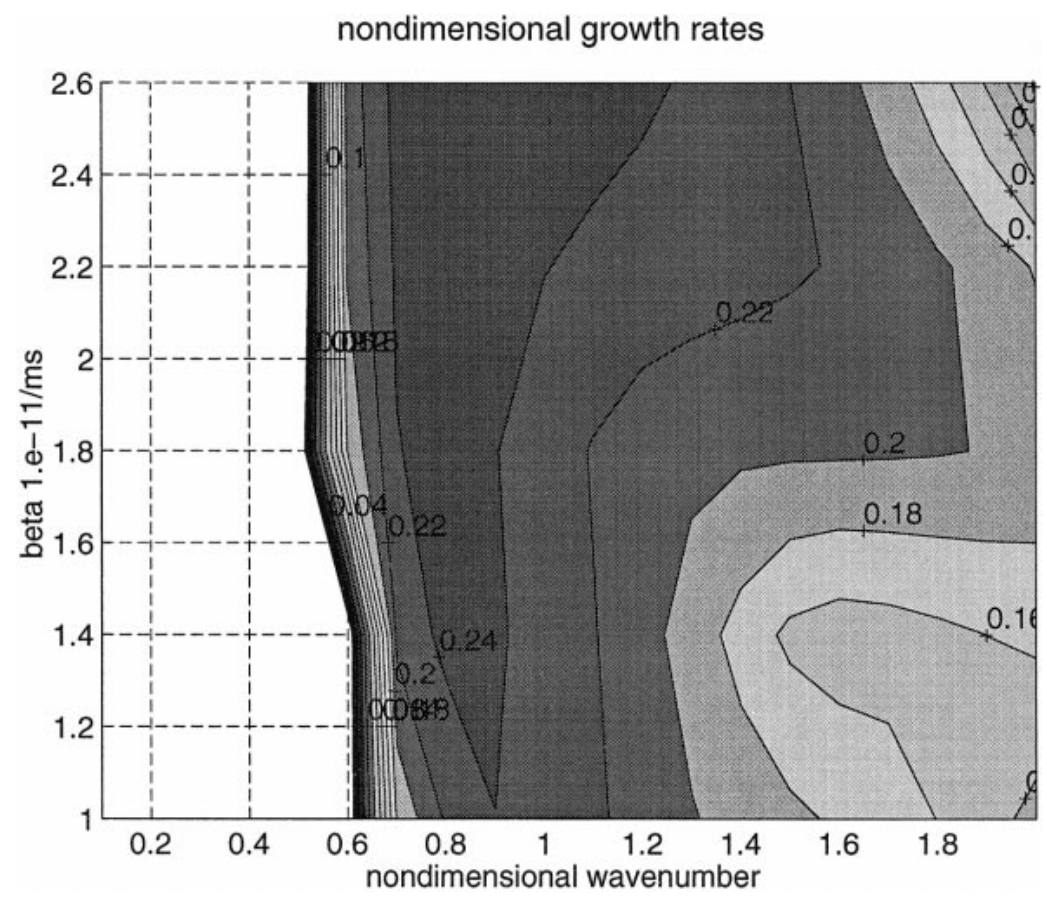

FIG. 5. Nondimensional growth rates when $\beta$ is increased from $1.0 \times 10^{-11} \mathrm{~m}^{-1} \mathrm{~s}^{-1}$ to $2.6 \times 10^{-11} \mathrm{~m}^{-1} \mathrm{~s}^{-1}$, from the model run using the staggered scheme for the 9-point half jet with $1 / D=20$ days and $u_{0}=-35 \mathrm{~m} \mathrm{~s}^{-1}$.

$\beta$ parameter study with the same parameters as Fig. 5. The nondimensional wavenumber at which the maximum nondimensional growth rate occurs decreases as $\beta$ is increased. Increasing $\beta$ stabilizes the shorter nondimensional wavenumbers.

The parameter studies using 3-point resolution are shown in Figs. 8 and 9. Figure 8 shows the nondimensional growth rates for the same parameters as Figs. 6 and 4 , except zonal winds as strong as $-65 \mathrm{~m} \mathrm{~s}^{-1}$ are considered to show the impact of the decreased resolution more clearly. All nondimensional wavenumbers greater than 1.4 or less than 0.5 have been stabilized. The nondimensional growth rates at a zonal wind of $-35 \mathrm{~m} \mathrm{~s}^{-1}$ have been reduced by a factor of 2 compared to the 5- and 9-point runs. The most unstable dimensional wavenumber decreases from a wave 7 in the 5and 9-point runs to a wave 5 (figure not shown). All waves become stable for $u_{0}$ less than $-30 \mathrm{~m} \mathrm{~s}^{-1}$ in magnitude. Figure 9 is the 3 -point $\beta$ parameter study with the same parameters as Figs. 5 and 7. This figure is consistent with the results of Fig. 8. Increasing $\beta$ stabilizes long and short waves, decreases the nondimensional growth rates and causes all waves to become stable for $\beta$ greater than $2.1 \times 10^{-11} \mathrm{~m}^{-1} \mathrm{~s}^{-1}$.

The stabilization of the waves due to inadequate resolution, illustrated by the scaling analysis at the beginning of this section, is typically thought to impact the stability of the waves only at the critical zonal mean wind. Figures 2-9 demonstrate that inadequate resolution impacts the stability of the waves for a large range of values for the zonal mean winds at the vertex of the jet and $\beta$. For example, Fig. 8 shows that the results from the scaling analysis - that the most unstable wave shifts to smaller wavenumbers and the growth rate of the most unstable wave decreases as the potential vorticity gradient at the jet decreases due to inadequate resolution-impacts the stability of the waves for zonal mean winds at the vertex of the jet up to $-65 \mathrm{~m} \mathrm{~s}^{-1}$, even though the critical zonal mean wind is $-30 \mathrm{~m} \mathrm{~s}^{-1}$.

In all of the studies without dissipation, the critical zonal wind for instability was the zonal wind at which the potential vorticity gradient changed sign. For all basic states that had a positive definite potential vorticity gradient, the basic state was stable. For all basic states with a change of sign in the potential vorticity gradient, the basic state was unstable. This was not true for the studies that had dissipation. When the potential vorticity gradient changed sign, the basic state did not become unstable. The magnitude of the potential vorticity gradient that is needed to make the dissipative study unstable is supercritical relative to the nondissipative study.

\section{Wave-mean flow integration results}

The wave-mean flow problem retains the nonlinear terms that modify the mean flow while neglecting the nonlinear terms that directly modify the waves. Therefore, the waves can only act to modify themselves indirectly through changes in the mean flow, since the 

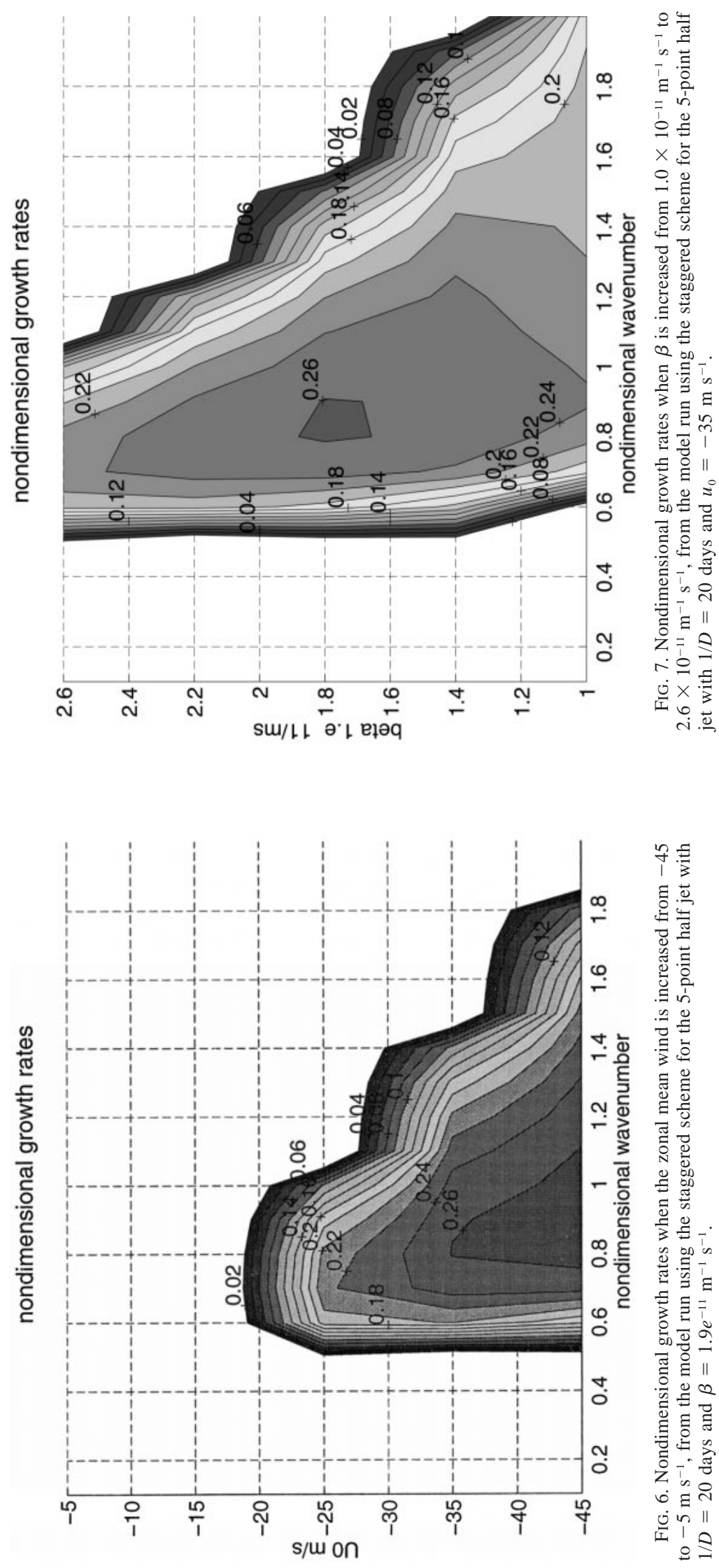

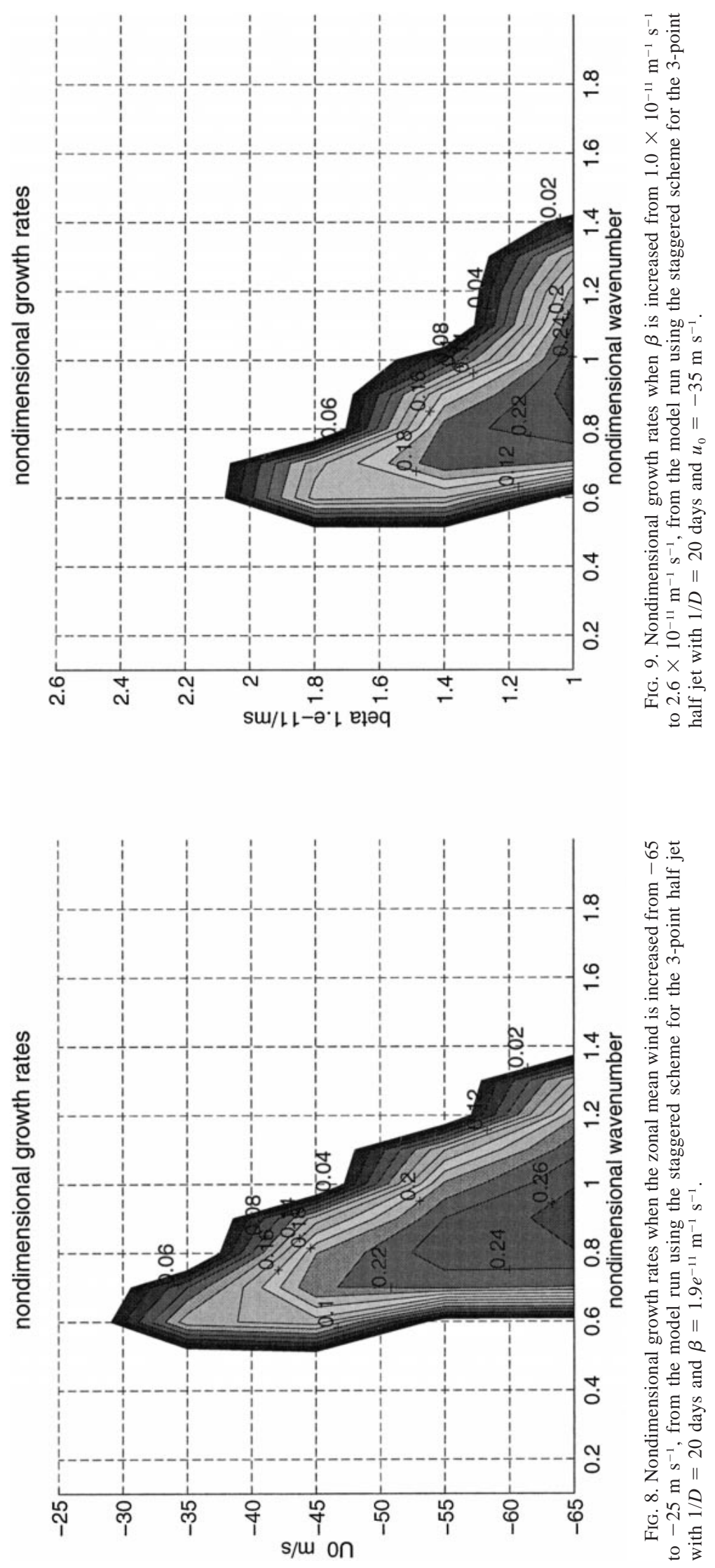
wave-mean flow system has no turbulent cascade of energy and enstrophy due to wave-wave interactions. Equation (1) is the equation that governs the waves. The equation that governs the changes in the mean flow is

$$
\frac{\partial \bar{q}}{\partial t}+\frac{\partial}{\partial y}\left(\overline{v^{\prime} q^{\prime}}\right)=-D\left(\bar{q}-q_{0}\right)
$$

where $q_{0}$ is the initial potential vorticity and $\bar{q}$ is the zonally averaged potential vorticity. The $D$ is the dissipation time constant. The wave-mean flow integrations are initialized with a small perturbation in waves $1-14$. Only waves $5,6,8,10,12,14$ are retained in the wave-mean flow integrations in order to illustrate the impact of resolution on the wave-mean flow dynamics and to allow for comparison with the results of Schoeberl and Lindzen (1984).

\section{a. Limits on channel-averaged wave enstrophy}

This section uses the results of Schoeberl and Lindzen (1984) to estimate the limits on the channel-averaged wave enstrophy as a function of resolution for the barotropic point jet problem. Schoeberl and Lindzen (1984) derived the limits on the channel-averaged wave enstrophy in the nondissipative system. Using conservation of enstrophy on constant pressure surfaces and assuming that the initial wave enstrophy is equal to zero, the channel-averaged wave enstrophy in the final state is

$$
\left\langle q_{f}^{\prime 2}\right\rangle=\left\langle\bar{q}_{i}^{2}\right\rangle-\left\langle\bar{q}_{f}^{2}\right\rangle,
$$

where \langle\rangle$=L^{-1} \int_{0}^{L}() d y$.

The final state of the wave-mean flow system is assumed to be defined by the Charney-Stern criterion for instability. In the final state, the wave-mean flow interactions have modified the mean flow such that all mean potential vorticity gradients are greater than or equal to zero. Following the work of Schoeberl and Lindzen (1984), the mean flow is assumed to be modified to a distance, $y_{c}$, from the jet maximum. For $0 \leq$ $y<y_{c}$, the mean potential vorticity is assumed to be unchanged from the initial state. Therefore, $y_{c}$ is calculated as the intersection between the initial and the final mean potential vorticity. The final state that minimizes the adjustment to the mean flow, and maximizes the channel-averaged wave enstrophy, is the state where the mean potential vorticity is equal to zero for $y_{c}<y$ $<L ; y_{c}$ is then found to be equal to $L-\Gamma / \beta$. The curvature of the jet in this final state is

$$
\frac{\partial^{2} \overline{u_{f}}}{\partial y^{2}}=\beta
$$

For this model the initial potential vorticity is

$$
q=f_{0}+\beta y+\Gamma, \quad y \leq L-\frac{\Delta y}{2},
$$

where $f_{0}+\beta y$ is equal to the Coriolis parameter. Letting
TABLE 2. Channel-averaged wave enstrophy for $\Gamma=1.4 \times 10^{-5}$ $\mathrm{m} \mathrm{s}^{-1}$, which corresponds to a zonal mean wind at the point of the jet of $-35 \mathrm{~m} \mathrm{~s}^{-1}$.

\begin{tabular}{ccc}
\hline \hline $\begin{array}{c}y_{c} \\
(\mathrm{pt})\end{array}$ & $(\mathrm{km})$ & $\begin{array}{c}\left\langle q_{f}^{\prime 2}\right\rangle \\
\left(10^{-11} \mathrm{~s}^{-2}\right)\end{array}$ \\
\hline 3 & 1875 & 0.2 \\
5 & 2188 & 1.6 \\
9 & 1719 & 1.9 \\
17 & 1797 & 1.9 \\
\hline
\end{tabular}

$n_{c}$ be the number of grid points from the point jet, at $y$ $=L$, to $y_{c}$,

$$
n_{c}=0.5+\left(\frac{\Gamma}{\beta \Delta y}\right),
$$

This gives an estimate of the channel-averaged wave enstrophy for a given number of gridpoints, $n$,

$$
\left\langle q_{f}^{\prime 2}\right\rangle=\frac{1}{n-1}\left[\sum_{i=1}^{n_{c}}\left(\frac{(2 i-1)}{2} \beta \Delta y-\Gamma\right)^{2}\right],
$$

where $n$ is a given number of evenly spaced grid points with a grid spacing of $\Delta y$. Table 2 lists the channelaveraged wave enstrophy for $\Gamma=1.4 \times 10^{-5} \mathrm{~s}^{-1}$, which corresponds to a zonal mean wind at the vertex of the jet equal to $-35 \mathrm{~m} \mathrm{~s}^{-1}$.

The analytic limit derived by Schoeberl and Lindzen (1984) for these parameters is

$$
\left\langle q_{f}^{\prime 2}\right\rangle=\frac{\Gamma^{3}}{3 L \beta}=1.9 \times 10^{-11} \mathrm{~s}^{-2} .
$$

The analytic value for $y_{c}=\Gamma / \beta$ is $1763 \mathrm{~km}$. Even though the wave enstrophy at the jet increases when the resolution is increased, due to the increase in the magnitude of the jump in the meridional potential vorticity gradient at the vertex of the jet, the channel-averaged wave enstrophy is independent of resolution. This is because the integrated jump in the potential vorticity gradients is independent of the resolution. The 3-point calculation for the channel-averaged wave enstrophy in the equilibrated state is an order of magnitude smaller than the analytic calculation. It is not clear how a model that does not resolve $y_{c}$ well will adjust to neutrality. The values for the channel-averaged wave enstrophy in the wave-mean flow neutral state, in units of $\mathrm{s}^{-2}$, are listed in Table 3.

The values for the channel-averaged wave enstrophy in the wave-mean flow neutral state, found by integration of (1) and (14), are shown in Table 3. Tables 2 and 3 together show that it is valid to assume that the initial jet structure is adjusted to neutrality by reducing the shear at the vertex of the jet such that the jump in the potential vorticity gradients is removed. The 3-point model is inadequate to resolve the channel-averaged wave entrophy of the higher-resolution runs. This is because the linear stability characteristics of the waves are inadequately resolved in the 3-point model with a 
TABLE 3. Values of the channel-averaged wave enstrophy in the wave-mean flow neutral state.

\begin{tabular}{cc}
\hline \hline$n$ & $\left\langle\begin{array}{c}\left.q_{f}^{\prime}\right\rangle \\
\left(10^{-11} \mathrm{~s}^{-2}\right)\end{array}\right.$ \\
\hline 3 & 0.6 \\
4 & 1.6 \\
5 & 1.7 \\
6 & 1.8 \\
7 & 1.9 \\
8 & 2.0 \\
9 & 2.1 \\
\hline
\end{tabular}

zonal mean wind at the vertex of the jet equal to -35 $\mathrm{m} \mathrm{s}^{-1}$, as shown in Fig. 10 and discussed in section $3 \mathrm{~b}$. Therefore, in this barotropic point jet problem, the adequacy of a model set up to resolve the channel-averaged wave-mean flow dynamics can be determined by accessing whether the the linear stability characteristics of the waves have been adequately resolved.

Figures 10, 11, and 12 show the partitioning of the channel-averaged wave enstrophy among waves $5,6,8$, $10,12,14$ in an undissipated system. Figure 10 is for a resolution of 9 points. All waves except wave 14 are unstable initially. Waves 5, 6, and 8 grow the fastest and modify the mean flow such that all of the waves become stabilized at approximately the same time. Since there is no exchange of energy or enstrophy among the waves in the wave-mean flow integration, the enstrophy of the waves changes very little after the waves have become stabilized. Figure 10 shows that there is not an equipartition of enstrophy in this neutral state. This is because of the large differences in the growth rates of the waves. An increase in resolution reduces this difference and causes the waves to grow at approximately the same rate. Therefore, enstrophy is distributed more equally as resolution is increased. Figure 11 shows the results of the 5-point wave-mean flow integration. The growth rates of waves 5,6 , and 8 are unaffected by the reduction in the resolution. The shorter waves 10 and 12 have been stabilized by the reduction in resolution. This is consistent with the stabilization of the shorter waves in the linear parameter studies (Figs. 4, 6, 8) when the resolution is decreased. Figure 12 shows the 3-point wave-mean flow integration. Only wave 5 is unstable for this resolution. The contribution to the channel-averaged wave enstrophy by the other waves is negligible. Figures 10, 11, and 12 are consistent with the linear parameter study which showed that the most unstable wave is shifted to longer wavelengths and the growth rate of the most unstable wave, for $u_{0}=-35 \mathrm{~m} \mathrm{~s}^{-1}$ and $\beta=1.9 e^{-11} \mathrm{~m}^{-1} \mathrm{~s}^{-1}$, is reduced by a factor of 2 when the resolution is decreased from 5 points to 3 points, while a decrease in resolution from 9 points to 5 points has little impact on either the wavelength of the most unstable wave or the growth rate of the most unstable wave (figure not shown).

Figures 13, 14, and 15 show the effect of adding dissipation. It is seen that the most unstable wave decays

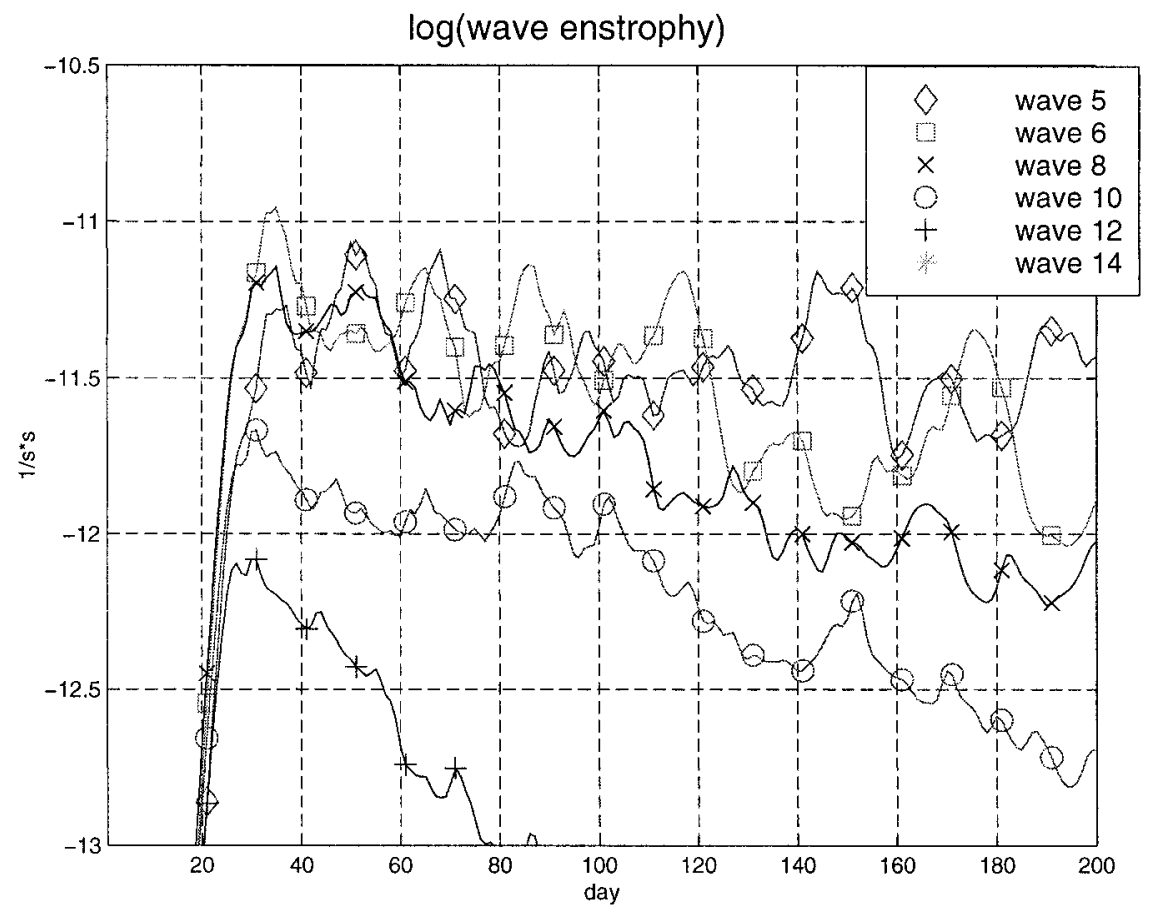

FIG. 10. The partitioning of the integrated wave enstrophy among waves $5,6,8,10,12,14$, from the 9-point wave-mean flow half-jet model run without dissipation with $u_{0}=-35 \mathrm{~m} \mathrm{~s}^{-1}$, $1 / D=0$ days, and $\beta=1.9 e^{-11} \mathrm{~m}^{-1} \mathrm{~s}^{-1}$. 

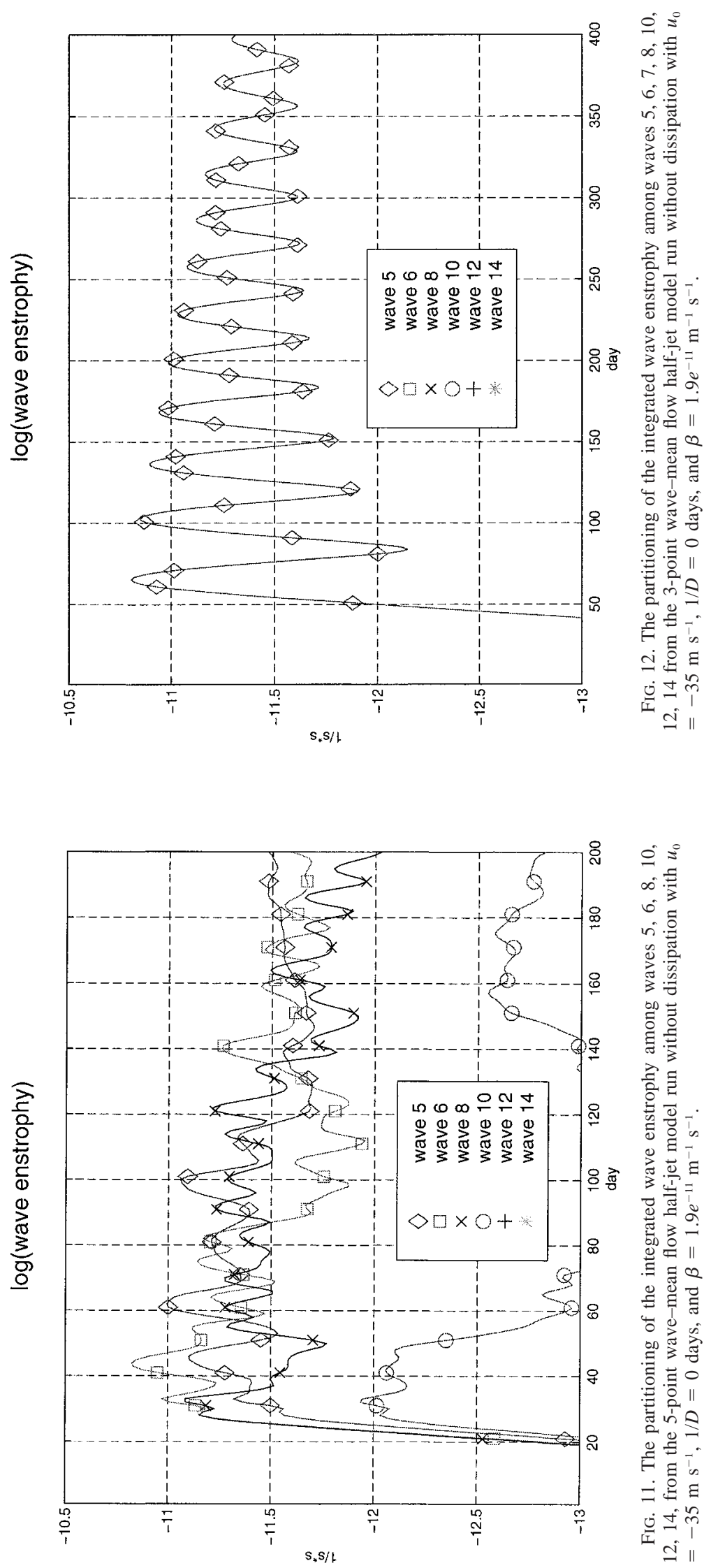

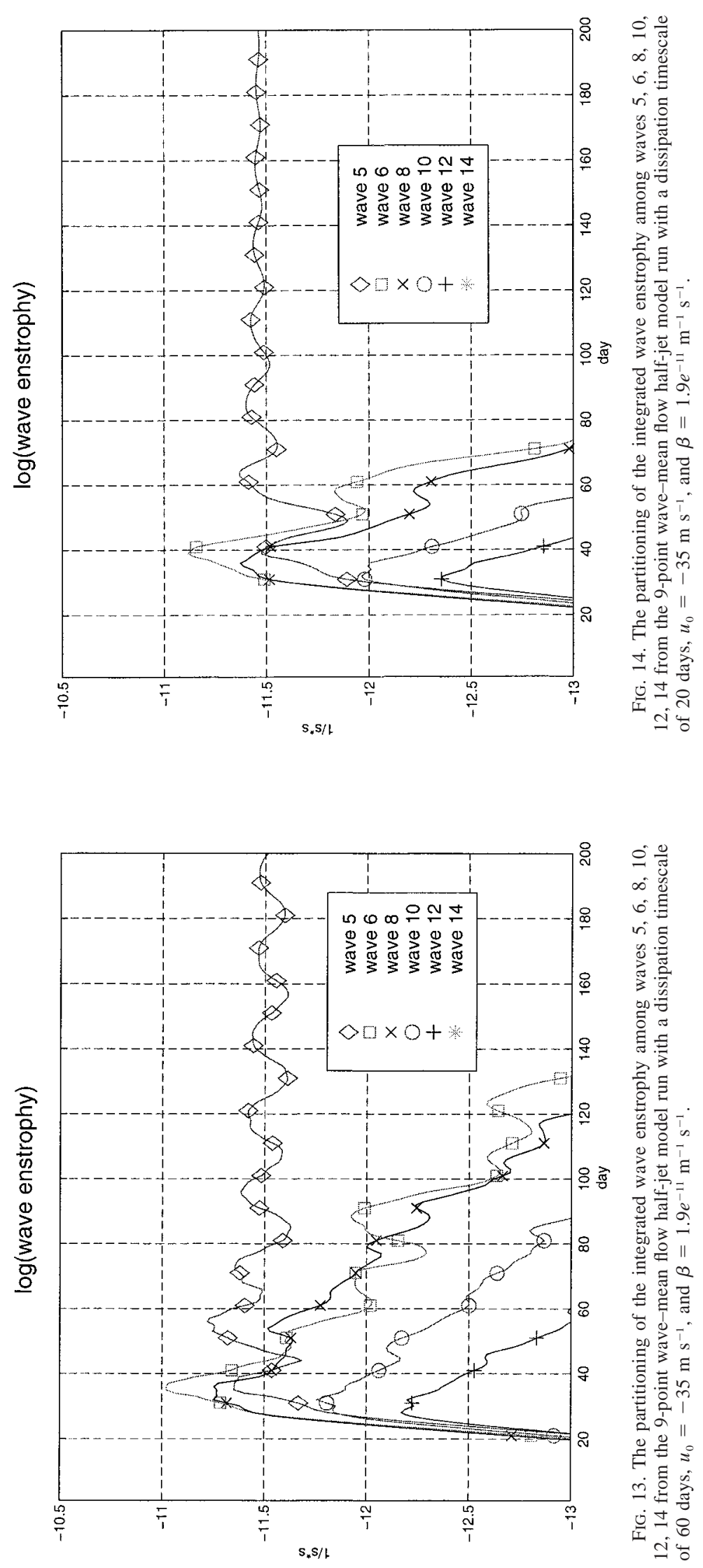


\section{$\log$ (wave enstrophy)}

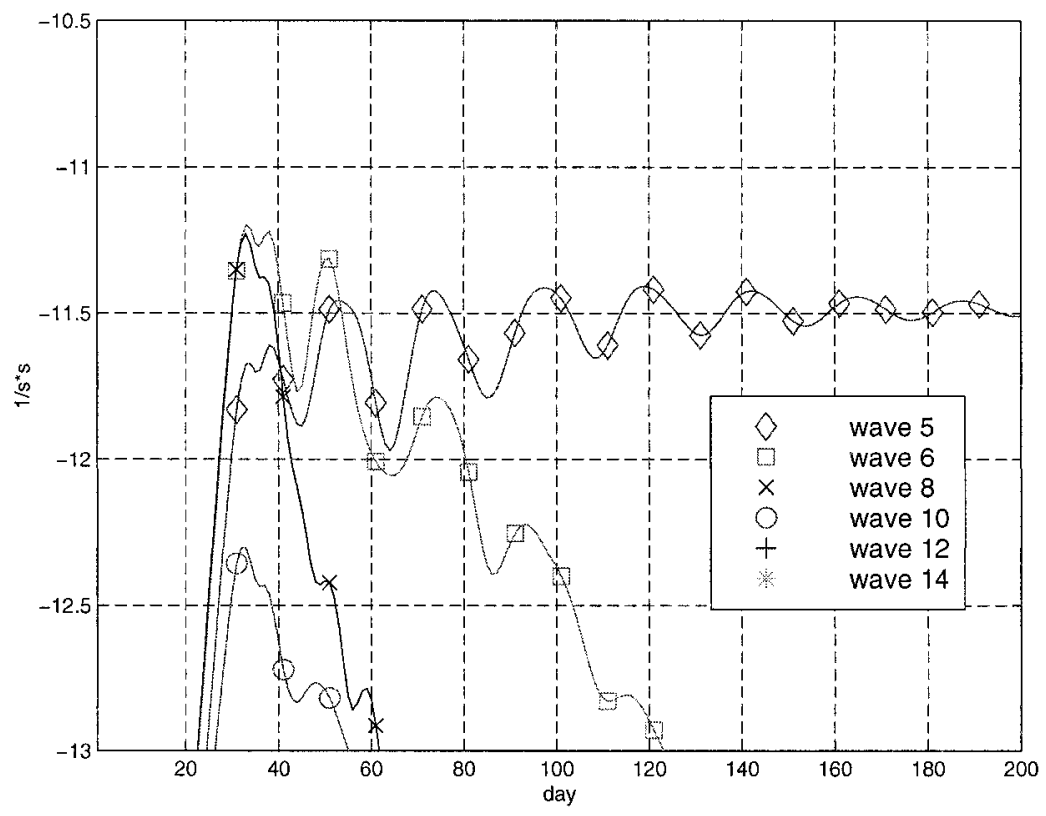

FIG. 15. The partitioning of the integrated wave enstrophy among waves 5, 6, 8, 10, 12,14 from the 5 -point wave-mean flow half-jet model run with a dissipation timescale of 20 days, $u_{0}=-35 \mathrm{~m} \mathrm{~s}^{-1}$, and $\beta=1.9 e^{-11} \mathrm{~m}^{-1} \mathrm{~s}^{-1}$.

away with the higher wavenumbers. Wave 5 grows slowly but becomes the dominant wave, even for a large dissipation time constant of 60 days. Therefore it is not only the growth rate of the waves that determines whether a wave will dissipate away.

When dissipation is added, the channel-averaged wave enstrophy of the neutral state is reduced from 1.5 $\times 10^{-11} \mathrm{~s}^{-2}$ to $0.4 \times 10^{-11} \mathrm{~s}^{-2}$. A comparison of Fig. 13 with Fig. 14 shows that this result is independent of the magnitude of the dissipation. The insensitivity of the channel-averaged wave enstrophy to the magnitude of the dissipation was also found in Schoeberl and Lindzen (1984). These results were found to be true for the 5-point study, Fig. 15. The 3-point study had a similar reduction of channel-averaged wave enstrophy, when the dissipation was added.

\section{b. Equilibrated states}

The meridional gradient of the potential vorticity at the jet is shown in Fig. 16a. The numbers in the legend show the time-averaged potential vorticity gradients at the jet for each of the runs. The 3- and 4-point runs are both significantly supercritical relative to the higherresolution runs, while the potential vorticity gradients from the 9- to 5-point models oscillate about the zero gradient line, which is the neutral state predicted by the Charney-Stern criterion. When dissipation is added to the system, the magnitude of the oscillations decreases and the equilibrated potential vorticity gradients become supercritical with a magnitude of approximately $-0.1 \beta$, independent of resolution and the strength of the dissipation (Fig. 17a). Considering the large differences in the initial potential vorticity gradients at the jet, which are shown at the beginning of the time series, the equilibrated potential vorticity gradients appear to be quite robust. Figures $16 \mathrm{~b}$ and $16 \mathrm{c}$ show that the potential vorticity flux convergence and potential vorticity flux at the jet oscillate about the zero line with oscillations of similar magnitude and timescales for all runs with greater than 3 points. The 3 -point run is seen to have oscillations on a much longer timescale than the other runs, but similar magnitude.

The time rate of change of the zonally averaged wave enstrophy was derived by Schoeberl and Lindzen (1984) to be

$$
\frac{1}{2} \frac{\partial\left(\overline{q^{\prime 2}}\right)}{\partial t}=-\left(\overline{v^{\prime} q^{\prime}}\right) \frac{\partial \bar{q}}{\partial y}-D\left(\overline{q^{\prime 2}}\right) .
$$

If the time rate of change of the zonally averaged wave enstrophy goes to zero, then the zonally averaged flux of the perturbation potential vorticity down the gradient of mean potential vorticity must balance the dissipation of the zonally averaged wave enstrophy. An increase in the zonally averaged wave enstrophy of the neutral state, or a decrease in the dissipation time constant, must be accompanied by either an increase in the magnitude of the mean potential vorticity gradient or an increase in the zonally averaged flux of perturbation potential vorticity. If the system is dissipationless and the meridional 

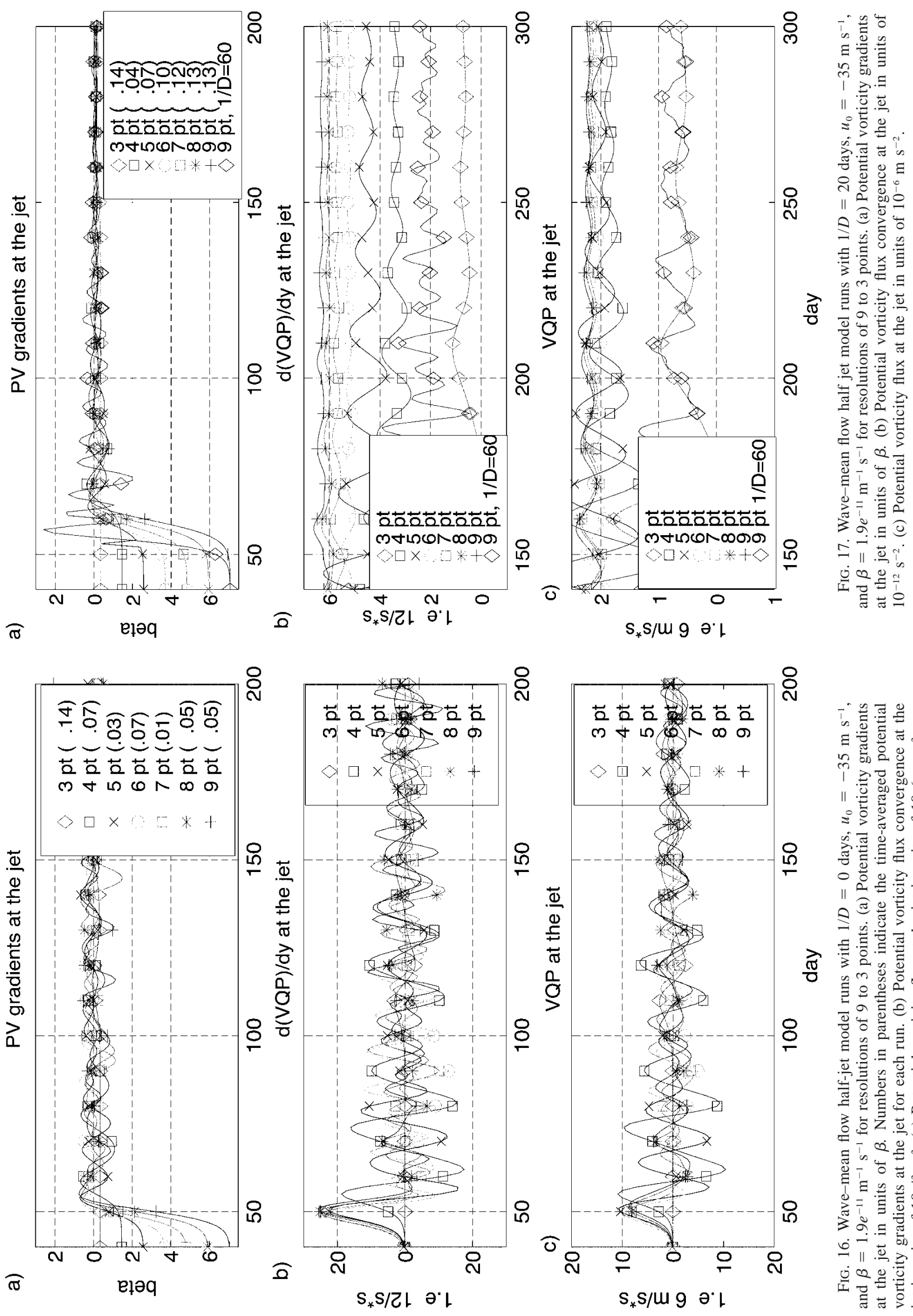

in

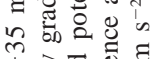

i.

11.0

월

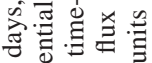

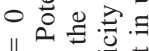

$\therefore$ @

㱐:

在

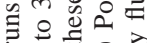

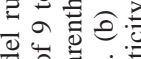

要范言

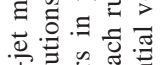

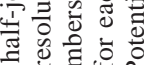

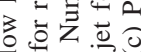

的里

का

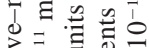

วัด

- 11.

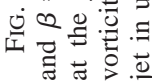


TABLE 4. Wave-mean flow values at the jet, where $u_{0}$ has been set equal to $-35 \mathrm{~m} \mathrm{~s}^{-1}$ and $D^{-1}$ has been set equal to 20 days.

\begin{tabular}{ccccc}
\hline \hline $\begin{array}{c}n \\
(\mathrm{pt})\end{array}$ & $\begin{array}{c}\overline{v^{\prime} q^{\prime}} \\
\left(10^{-6} \mathrm{~m} \mathrm{~s}^{-2}\right)\end{array}$ & $\begin{array}{c}\partial \bar{q} / \partial y \\
\left(10^{-12} \mathrm{~m}^{-1} \mathrm{~s}^{-1}\right)\end{array}$ & $\begin{array}{c}\overline{q^{\prime 2}} \\
\left(10^{-12} \mathrm{~s}^{-2}\right)\end{array}$ & $\begin{array}{c}\Gamma_{f} \\
\left(10^{-6} \mathrm{~s}^{-1}\right)\end{array}$ \\
\hline 3 & 0.6 & -1.4 & 1.3 & 12.8 \\
4 & 1.9 & -0.4 & 1.2 & 8.1 \\
5 & 2.0 & -0.7 & 2.2 & 6.2 \\
6 & 2.1 & -1.0 & 3.2 & 5.0 \\
7 & 2.1 & -1.2 & 3.9 & 4.2 \\
8 & 2.2 & -1.3 & 4.3 & 4.1 \\
9 & 2.2 & -1.3 & 4.6 & 3.2 \\
\hline
\end{tabular}

gradient of the potential vorticity is not equal to zero, then the potential vorticity flux goes to zero as the time rate of change of the wave enstrophy goes to zero.

Figure $17 \mathrm{~b}$ shows the zonally averaged potential vorticity flux convergence at the jet for the 3- to 9-point model runs. This figure shows that, owing to the increase in the potential vorticity gradient at the jet as resolution is increased, the potential vorticity flux convergence systematically increases as the resolution is increased. Since the initial and final potential vorticity gradients were shown to be independent of the strength of the dissipation, the potential vorticity flux convergence decreases by a factor of 3 when the dissipation timescale is increased by a factor of 3 .

Figure $17 \mathrm{c}$ shows the zonally averaged flux of perturbation potential vorticity at the jet for the 9- to 3-point resolution models with dissipation. This figure shows that the potential vorticity flux at the jet is robust for all runs with 4 points or more. The 3-point run has potential vorticity flux at the jet that is a factor of 3 smaller than the potential vorticity flux at the jet for the 9- to 4-point resolution runs. This is consistent with the underestimate of the channel-averaged enstrophy from Table 3. Increasing the dissipation time constant by a factor of 3 decreases the potential vorticity flux at the jet by a factor of 3 .

The relationships described above are illustrated in Table 4 . Table 4 lists the wave-mean flow values at the jet, where $u_{0}$ has been set equal to $-35 \mathrm{~m} \mathrm{~s}^{-1}$ and $D^{-1}$ has been set equal to 20 days. This table clearly shows that the wave enstrophy and shear at the jet are a function of resolution. This table shows that the model equilibrates to a state where the potential vorticity gradients at the jet are independent of resolution. Assuming that the equilibrated potential vorticity gradients at the jet are robust and that the wave enstrophy at the jet decreases as the resolution is decreased, Eq. (21) indicates that the potential vorticity flux at the jet will also decrease as the resolution is decreased. This relationship is seen to break down in the 3-point model where the underestimate in the magnitude of the potential vorticity gradients is balanced by an increase in the potential vorticity flux.
TABLE 5. Critical shears and temperature gradients for the linear baroclinic model, which have been calculated from the values of the linear barotropic model in Table 1.

\begin{tabular}{cccc}
\hline \hline $\begin{array}{c}n \\
(\mathrm{pt})\end{array}$ & $\begin{array}{c}u_{c} \\
\left(\mathrm{~m} \mathrm{~s}^{-1}\right)\end{array}$ & $\begin{array}{c}\Gamma_{c} \\
\left(10^{-4} \mathrm{~s}^{-1}\right)\end{array}$ & $\begin{array}{c}\partial \overline{\theta_{c}} / \partial y \\
\left(10^{-3} \mathrm{~K} \mathrm{~km}^{-1}\right)\end{array}$ \\
\hline 3 & 2.6 & 3.5 & -1.0 \\
5 & 1.3 & 1.8 & -0.5 \\
9 & 0.7 & 0.9 & -0.3 \\
\hline
\end{tabular}

\section{Baroclinic interpretation of barotropic results}

The linearized barotropic jet is analogous to the linearized Boussinesq Charney problem. Therefore, the results of the linear barotropic jet can be extended to the linear Boussinesq Charney stability problem. These results are that the most unstable wavenumber will shift to lower wavenumbers when the resolution is decreased. The growth rates of the waves will decrease and the shortwaves will become stabilized as the resolution is decreased. The critical mean zonal wind for instability will double when the resolution is reduced by half. A reduction in resolution will stabilize the waves by increasing the critical zonal mean wind for instability.

The barotropic problem assumes that the zonal wind is symmetric about the vertex of the jet. This means that the shear and the zonal mean potential vorticity are equal to zero at the vertex of the jet. In the analogous baroclinic problem the vertex of the jet corresponds to the lower boundary. This lower boundary in the baroclinic problem represents the surface of the earth. The meridional shear in the barotropic problem corresponds to the vertical shear in the baroclinic problem. By the thermal wind relation, the vertical shear is proportional to the meridional temperature gradient. In the linear Boussinesq Charney stability problem a delta function in the potential vorticity gradients very close to the lower rigid boundary is equivalent to temperature gradients along this rigid boundary, making this problem relevant to the earth's atmosphere. This classic result was first pointed out by Bretherton (1966). The critical shear is the shear necessary to eliminate the negative potential vorticity gradients in the model and is expressed as

$$
\Gamma_{c}=\frac{\beta N^{2}}{f_{0}^{2}} \frac{H}{(n-1)} .
$$

Here $N^{2}$ has been set equal to $1 \times 10^{-4} \mathrm{~s}^{-2} ; f_{0}$ is the Coriolis parameter and is set equal to $1 \times 10^{-4} \mathrm{~s}^{-1} ; H$ is the vertical scale height of the troposphere and is set equal to $7.4 \mathrm{~km} ; n$ is the resolution of the model. The critical zonal wind, assuming a constant initial shear, is expressed as $\Gamma_{c} H$. The critical shears and temperature gradients for the linear baroclinic model that have been calculated from the values of the linear barotropic model in Table 1 are listed in Table 5.

The temperature gradient is calculated using the equation 
TABLE 6. Characteristics of the baroclinic equilibrium state calculated from the values of the barotropic wave-mean flow neutral state.

\begin{tabular}{cccc}
\hline \hline $\begin{array}{c}n \\
(\mathrm{pt})\end{array}$ & $\begin{array}{c}v^{\prime} q^{\prime} \\
\left(10^{-5} \mathrm{~m} \mathrm{~s}^{-2}\right)\end{array}$ & $\begin{array}{c}\Gamma_{f} \\
\left(10^{-4} \mathrm{~s}^{-1}\right)\end{array}$ & $\begin{array}{c}\partial \overline{\theta_{c}} / \partial y \\
\left(10^{-3} \mathrm{~K} \mathrm{~km}^{-1}\right)\end{array}$ \\
\hline 3 & 0.7 & 3.7 & -1.1 \\
4 & 2.1 & 2.3 & -0.6 \\
5 & 2.3 & 1.8 & -0.5 \\
6 & 2.5 & 1.5 & -0.5 \\
7 & 2.5 & 1.2 & -0.4 \\
8 & 2.6 & 1.2 & -0.4 \\
9 & 2.6 & 0.9 & -0.3 \\
\hline
\end{tabular}

$$
\frac{\partial \bar{\theta}}{\partial y}=-\frac{f_{0} \theta_{0}}{g} \frac{\partial \bar{u}}{\partial z}
$$

These temperature gradients correspond to a temperature difference across a distance of $40^{\circ}$ : $-4.6 \mathrm{~K}$ for $n$ $=3,-2.3 \mathrm{~K}$ for $n=5$, and $-1.2 \mathrm{~K}$ for $n=9$. These values are for an unforced and dissipationless model. The continuous formulation of the point jet has no critical shear or critical temperature gradient. The critical shear that is calculated in Table 5 is strictly due to the truncation of the model. As the resolution is increased the critical shear is reduced. For a resolution of 3 points, which is equivalent to the two-level model, the critical shear and critical temperature gradients can be of the order of observed midlatitude surface shears and temperature gradients. For example, observations (Peixoto and Oort 1992) yield an annual mean temperature gradient of $-4 \times 10^{-3} \mathrm{~K} \mathrm{~km}^{-1}$ and an annual mean zonal wind shear of $7 \times 10^{-4} \mathrm{~s}^{-1}$, for the Northern Hemispheric mean at approximately $800 \mathrm{hPa}$.

The linearized barotropic jet is analogous to the linearized Boussinesq Charney problem. This equivalence does not hold when the nonlinear terms become large enough to modify the mean state or when meridional scales are introduced into the problem. Therefore the barotropic wave-mean flow results can only be used to suggest how resolution will modify the wave-mean flow dynamics of the baroclinic problem.

The characteristics of the baroclinic equilibrium state calculated from the values of the barotropic wave-mean flow neutral state listed in Table 4 , for $D^{-1}=20$ days, are listed in Table 6, where

$$
\begin{gathered}
\partial / \partial y=-\frac{f_{0}}{N} \partial / \partial z \quad \text { and } \\
{\overline{v^{\prime} q^{\prime}}}_{\text {baroclinic }}=\frac{f_{0}^{2}}{N^{2}} \frac{L^{2}}{H^{2}} \overline{v^{\prime} q^{\prime}} \text { barotropic }
\end{gathered}
$$

These results show that if the resolution is adequate to resolve the analytic linear growth rates (model runs with points greater than 5) then the potential vorticity gradients and flux in the equilibrated state will be independent of resolution. These robust potential vorticity gradients at the jet result in shear and meridional tem- perature gradients that increase in magnitude as the resolution decreases.

\section{Summary}

This study clearly shows that resolution can impact in two ways the numerical simulation of barotropic instability. The first impact is due to an increase in the potential vorticity gradients at the jet when the resolution is increased. It is found that this results in wave enstrophy at the jet that increases as the resolution is increased, even though the channel-averaged wave enstrophy is shown to be conserved. Therefore, even though the dynamics at the jet change as a function of resolution, there are channel-averaged properties that are conserved. This would not be true if a larger zonal mean wind had been used in the wave-mean flow integrations since the 3-point integration becomes stable for zonal mean winds less than $-30 \mathrm{~m} \mathrm{~s}^{-1}$ in magnitude. When the resolution of the model is adequate to resolve the linear analytic growth rates, the mean potential vorticity structure and potential vorticity flux in the equilibrated state are independent of resolution. This is consistent with momentum fluxes and shear at the jet that are dependent upon the resolution of the model.

The resolution of the model is also found to impact the wave-mean flow equilibrated state when the linear analytic growth rates are inadequately resolved. This result is shown to be dependent upon the values of the initial zonal mean wind at the jet and $\beta$. A linear parameter study demonstrates that not only is there a critical zonal mean wind for stability but there is a large range of mean zonal winds and $\beta$ where the growth rates of the waves are underestimated because of inadequate resolution. For the values of the zonal mean wind and $\beta$ chosen for the wave-mean flow runs, the dynamics of the 3-point model are clearly modified by the inadequate resolution. It is found that the resolution of the model has a significant impact on the partitioning of the integrated wave enstrophy. It is shown that the growth rates of both the long waves and the short waves are reduced as the resolution is decreased, resulting in integrated wave enstrophy that is not partitioned equally among the waves.

This study also shows that there are many aspects of the wave-mean flow dynamics that are independent of resolution. Runs that had no dissipation equilibrate to the state predicted by the Charney-Stern criterion; the mean state was adjusted to eliminate the change of sign in the initial potential vorticity gradients. Runs with dissipation have equilibrated states that are supercritical to the Charney-Stern criterion. These results are consistent with previous studies of the barotropic point jet (Schoeberl and Lindzen 1984; Schoeberl and Nielsen 1986). A reduction in resolution does not change the basic result found by Schoeberl and Lindzen (1984) that a basic state that is neutral to the most unstable wave 
and to wavelengths shorter than the most unstable wave is still unstable to longer waves.

The impact of resolution on the numerical simulation of barotropic instability can be directly extended to the purely baroclinic problem due to the mathematic equivalence between the barotropic easterly jet stability problem and the linear Boussinesq Charney problem (Lindzen et al. 1983). The impact of resolution on the wavemean flow equilibrated states in the barotropic problem suggests that the wave-mean flow equilibrated states in the baroclinic problem will also be dependent upon resolution. Using reasonable values for the initial zonal mean wind and $\beta$ and transforming meridional gradients in the barotropic problem to be consistent with vertical gradients in the baroclinic problem, the critical temperature gradients and vertical shears for instability in the two-level formulation of the model are found to be of the order of observations. This result is purely a function of the resolution and is thus fortuitous since all vertical shears and temperature gradients are unstable in the continuous problem.

This study has shown that coarse-resolution baroclinic models can be used to study wave-mean flow dynamics, in a channel-averaged sense, if the linear stability characteristics of the waves are adequately resolved. This study further suggests that the adequacy of these coarse-resolution models, used in studies such as Stone and Branscome (1992), Zhou and Stone (1993), and Held (1978b), can be determined by testing whether the linear stability characteristics of the waves are resolved in each case.

Acknowledgments. This work has been supported by the National Aeronautics and Space Administration under Grant NAG5-4880 to MIT. R. S. Lindzen's efforts were supported by Grants ATM 9421195 from NSF, DEFG02-93ER61673 from DOE, and NAG5-6304 from NASA.

\section{REFERENCES}

Bretherton, F. P., 1966: Critical layer instability in baroclinic flows. Quart. J. Roy. Meteor. Soc., 92, 325-334.
Gutowski, W. J., Jr., 1985: Baroclinic adjustment and midlatitude temperature profiles. J. Atmos. Sci., 42, 1733-1745.

—, L. E. Branscome, and D. A. Stewart, 1989: Mean flow adjustment during life cycles of baroclinic waves. J. Atmos. Sci., 46, 1724-1737.

Held, I. M., 1978a: The vertical scale of an unstable wave and its importance for eddy heat flux parameterizations. J. Atmos. Sci., 35, 572-576.

_ 1978b: The tropospheric lapse rate and climatic sensitivity: Experiments with a two-level atmospheric model. J. Atmos. Sci., 35, 2083-2098.

— mospheric model designed for climatic sensitivity experiments. J. Atmos. Sci., 35, 206-229.

Lindzen, R. S., 1993: Dynamics in Atmospheric Physics. Cambridge University Press, $310 \mathrm{pp}$.

— maximum growth rate of baroclinic instabilities. J. Atmos. Sci., 37, 1648-1654.

— and a new parameterization of global heat transport. Mon. Wea. Rev., 108, 2064-2079.

— A. J. Rosenthal, and B. F. Farrell, 1983: Charney's problem for baroclinic instability applied to barotropic instability. J. Atmos. Sci., 40, 1029-1034.

Nielsen, J. E., and M. R. Schoeberl, 1984: A numerical simulation of barotropic instability. Part II: Wave-wave interaction. J. Atmos. Sci., 41, 2869-2881.

Peixoto, J. P., and A. H. Oort, 1992: Physics of Climate. American Institute of Physics, $520 \mathrm{pp}$.

Phillips, N. A., 1954: Energy transformation and meridional circulations associated with simple baroclinic waves in a two-level quasi-geostrophic model. Tellus, 6, 273-286.

Schoeberl, M. R., and R. S. Lindzen, 1984: A numerical simulation of barotropic instability. Part I: Wave-mean flow interaction. $J$. Atmos. Sci., 41, 1368-1379.

— instability. Part III: Wave-wave interaction in the presence of dissipation. J. Atmos. Sci., 43, 1045-1050.

Stone, P. H., 1978: Baroclinic adjustment. J. Atmos. Sci., 35, 561571.

_ eddy regimes. J. Atmos. Sci., 49, 355-367.

Thompson, P. D., 1961: Numerical Weather Analysis and Prediction. Macmillan, $170 \mathrm{pp}$.

Zhou, S., and P. H. Stone, 1993: The role of large-scale eddies in the climate equilibrium. Part II: Variable static stability. J. Climate, 6, 1871-1881. 